\title{
Afterdepolarizations Promote the Transition From Ventricular Tachycardia to Fibrillation in a Three-Dimensional Model of Cardiac Tissue
}

\author{
Takashi Ashihara, MD; Takenori Yao, MD; Tsunetoyo Namba, MD*; \\ Ayaka Kawase, MD**; Takanori Ikeda, MD**; \\ Kazuo Nakazawa, $\mathrm{PhD}^{\dagger}$; Makoto Ito, MD
}

\begin{abstract}
Recent experimental results regarding the action potential duration restitution curve have explained the transition from ventricular tachycardia (VT) to fibrillation (VF) in terms of spiral wave (SW) meandering and breakup. However, it remains unclear whether VF always has a steep restitution curve. The present study was designed to test the hypothesis that afterdepolarizations occur at excitable gaps during VF and affect the SW dynamics, even if the restitution curve is gentle. Homogeneous and isotropic 3-dimensional tissue was simulated with a LRd model. Because of the gentle restitution curve, it was not expected that SW instabilities would occur in this condition. In the tissue, a stationary SW reentry was initially observed; however, afterdepolarizations erupted from the excitable gap near the SW tip, and the SW then meandered widely. Following that, afterdepolarizations erupted far from the SW tip, resulting in SW breakup. In this manner, the wave dynamics degenerated into a chaotic state within a few seconds. Furthermore, not only triggered activity but also subthreshold afterdepolarizations were found to cause SW instabilities. These results suggest that afterdepolarizations may play an important role in the transition to VF and that the mechanism is independent of restitution properties. (Circ J 2002; 66: 505-510)
\end{abstract}

Key Words: Afterdepolarizations; Restitution; Spiral wave; Ventricular fibrillation; Ventricular tachycardia

$\mathbf{R}$ ecent experimental studies have shown that the spiral or scroll wave (SW) is one of the important mechanisms of ventricular tachyarrhythmias!,2 Clinical observations have suggested that ventricular fibrillation (VF) is usually preceded by ventricular tachycardia (VT), and some experiments and simulation studies have suggested that the mechanism of the transition from VT to VF is based on SW meandering ${ }^{1,2}$ and/or breakup4-7 The Restitution Hypothesis ${ }^{4,6,7}$ has explained the mechanism of the SW breakup caused by the interaction between the wavefront and the wavetail: A steep slope $(>1)$ of the action potential duration (APD) restitution curve results in spontaneous SW breakup, and a gentle slope $(<1)$ results in SW stability.

Afterdepolarizations are defined as oscillations in the myocardial cell membrane potential that follow the upstroke of an action potential. In 1962, Reiter ${ }^{8}$ reported that digitalis administration or high calcium concentration produced a series of spontaneous afterdepolarizations (aftercontractions) in guinea pig papillary muscles, and that these afterdepolarizations were induced by a greater rate of stimulation. Early and delayed afterdepolarizations (EADs

(Received December 5, 2001; revised manuscript received January 23, 2002; accepted January 31, 2002)

First Department of Internal Medicine, Shiga University of Medical Science, Otsu, *Department of Medical Technology, Kagawa Prefectural College of Health Sciences, Kita, **Third Department of Internal Medicine, Toho University School of Medicine, Tokyo and National Cardiovascular Center Research Institute, Suita, Japan Mailing address: Takashi Ashihara, MD, PhD, First Department of Internal Medicine, Shiga University of Medical Science, Seta Tsukinowa-cho, Otsu 520-2192, Japan. E-mail: ash@belle.shigamed.ac.jp and DADs, respectively) induce triggered activity (TA) ${ }^{9}$ that can result in the induction of cardiac arrhythmias ${ }^{10-15}$ The EADs are classified into phase-2 and phase-3 EADs. The phase-2 EADs can be induced experimentally by various conditions, even in the absence of calcium overload. On the other hand, according to the present view, the DADs and phase-3 EADs are associated with fast rates and calcium overload conditions, and the phase-3 EADs are considered to share a common mechanism with DADs; ${ }^{16,17}$ that is, calcium ions are spontaneously released (calcium-inducedcalcium-release) from the overloaded sarcoplasmic reticulum (SR) ${ }^{18,19}$ and this generates an intracellular calcium transient that activates the inward current of sodiumcalcium exchanger (INaCa) and the nonspecific calciumactivated current $(\operatorname{Ins}(\mathrm{Ca}))$ to depolarize the myocardial cell membrane ${ }^{16}$ In addition, the enhancement of inward $\mathrm{INaCa}$ and that of inward $\operatorname{Ins}(\mathrm{Ca})$ in response to calcium overload, and their contributions to membrane depolarization, are comparable.

However, there are a number of points regarding the mechanisms of the transition from VT to VF that have not been clarified, and it remains unclear whether VF always has a steep APD restitution curve. Moreover, it is unknown whether the DADs and phase-3 EADs occur while a SW rotates. Accordingly, we hypothesized that these afterdepolarizations occur at excitable gaps during SW reentry and, even if the maximum slope of the APD restitution curve is gentle $(<1)$, the afterdepolarizations will affect the SW dynamics, such as meandering and wave breakup. To confirm this hypothesis, we studied the behavior of SW reentry in 3-dimensional (3-D) computer simulations using a realistic mammalian ventricular action potential model. 


\section{Methods}

\section{Simulated Myocardial Tissue}

We used the LRd (Luo-Rudy-2) ventricular action potential model ${ }^{16,20}$ without any modifications as the myocardial unit in this study. The validation of the LRd model has been addressed in previous reports 16,20 The model includes the following currents: fast sodium (INa), L-type calcium (ICaL), time-dependent potassium (IK), time-independent potassium (IK1), plateau potassium (IKp), sodium-calcium exchanger (INaCa), sodium - potassium pump (INaK), nonspecific calcium-activated (Ins $(\mathrm{Ca}))$, sarcolemmal calcium pump (Ip $(\mathrm{Ca}))$, calcium background (ICa,b), sodium background (INa,b), and the time-independent, purely voltagedependent current (Iv). Furthermore, this model incorporates calcium release (Irel) and uptake (Iup) by the SR, the translocation of calcium between 2 compartments in the SR (the network SR and the junctional $\mathrm{SR}^{21}$ ) (Itr), and the buffering processes in the SR and the myoplasm; therefore, it is possible to simulate the behavior of cells under calcium overload conditions, and the related arrhythmogenic activity, such as EADs, DADs, and TA, of the myocardial cells.

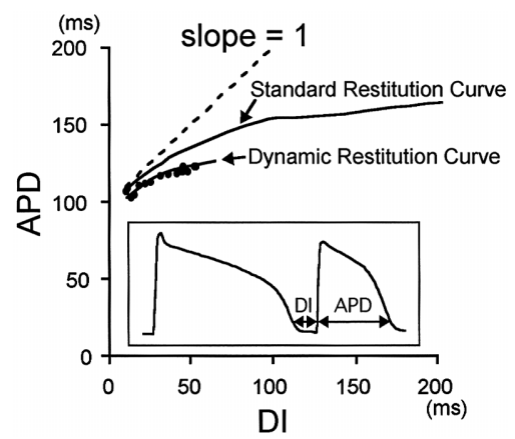

Fig 1. The standard action potential duration (APD) restitution curve (upper solid curved line) and dynamic APD restitution curve (lower solid curved line with black dots). The preceding diastolic interval (DI) is plotted along the horizontal axis, and the APD on the vertical axis. The dotted oblique line represents slope $=1$. The maximum slopes of those restitution curves were below 1 .
Next, we composed a homogeneous and isotropic 3-D myocardial tissue model, $6.0 \times 6.0 \times 1.0 \mathrm{~cm}(4,500,000$ units). The values of the parameters used to establish the electrical conductivity of the tissue were: the spatial discretization step in all directions was $0.02 \mathrm{~cm}$, the time discretization step was $0.01 \mathrm{~ms}$, the membrane capacitance was $1.0 \mathrm{~F} / \mathrm{cm}^{2}$, the conductivity between the myocardial units was $1.0 \mathrm{mS} / \mathrm{cm}$, and the surface-to-volume ratio was $1,800 / \mathrm{cm}$. The conduction velocity of a planar wave was calculated as 0.387 $\mathrm{m} / \mathrm{s}$. The tissue borders were set to non-flux Neumann boundary conditions. The SWs were generated by the S1S2 method, where a premature stimulus in the shape of a part of a globe was administered when a refractory tail of a planar wave had just passed through the center of the simulated tissue.

\section{APD Restitution Curve and Simulated ECG}

The standard APD restitution curve was obtained by premature stimuli after a pacing protocol performed 8 times at $500 \mathrm{~ms}$. The APD and the restitution curve were measured in one myocardial unit at a simulated temperature of $37^{\circ} \mathrm{C}$ with a pacing pulse duration of $5 \mathrm{~ms}$ and a pacing strength twice the diastolic threshold. The dynamic APD restitution curve was obtained during fibrillatory conduction in the 3-D myocardial tissue model.

The simulated electrocardiograms (ECGs) were calculated from the signals obtained at a unipolar electrode located $3.0 \mathrm{~cm}$ above the center of the epicardial (front) tissue surface. The equation used for the simulated ECG was described in our previous simulation study? 2

\section{Computations}

All the computations were performed on an NEC SX4/16 supercomputer (16GFLOPS; NEC, Tokyo, Japan). We used the forward Euler method for the integration of the LRd equations. It took about $50 \mathrm{~h}$ on the supercomputer to process a typical simulation of $3,000 \mathrm{~ms}$ of real time for the 3-D tissue. The convergence of the results for each simulation period was tested by repeating some simulations with half of the spatial and time discretization steps. We confirmed that decreasing these discretization steps did not significantly affect the simulation results.

\section{A}
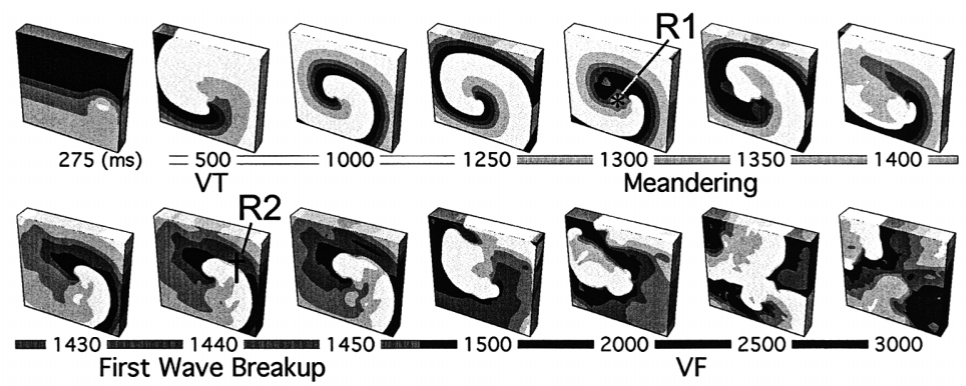

B
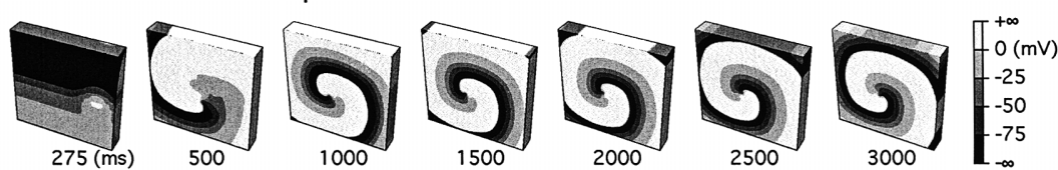

c

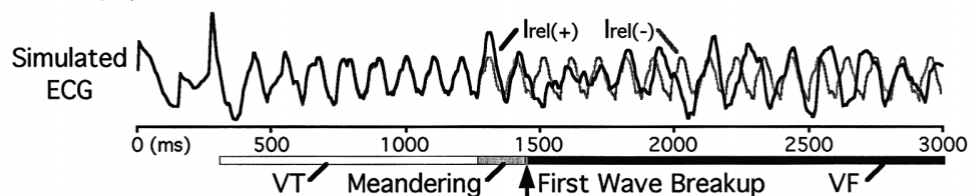

Fig 2. (A) Consecutive snapshots of the transmembrane potential maps during spiral wave $(\mathrm{SW})$ reentry in 3-dimensional cardiac tissue with the original LRd model. The excitation waves are displayed as a gray scale according to the potential (see gray scale bar). Asterisk (R1) and dagger (R2) represent the location of units recording some cellular level data (see Fig 4). (B) Consecutive snapshots of the transmembrane potential maps during SW reentry in the same tissue with the LRd model modifying the calcium ion release from the junctional sarcoplasmic reticulum to the myoplasm (Irel). The excitation waves are displayed as a gray scale according to the potential (see gray scale bar). (C) Simulated electrocardiograms (ECGs) from the transmembrane potential maps during the SW reentries. Black solid [Irel(+); original model] and shaded [Irel(-); modified model] lines represent the simulated ECGs of (A) and (B), respectively. 


\section{Results}

\section{APD Restitution Curve of the LRd Model}

Fig 1 shows the APD restitution curve obtained from the LRd model. We found that the maximum slopes of the APD restitution curves were gentle and less than 1. According to the Restitution Hypothesis, 4,7 it was not expected that SW meandering and/or breakup would occur under these myocardial conditions.

\section{Simulations in 3-D Tissue}

Fig 2A shows consecutive snapshots of the transmembrane potential maps during SW reentry. After the S2 stimulus $(275 \mathrm{~ms})$, a stationary SW reentry $(500-1,250 \mathrm{~ms})$ was observed. However, the afterdepolarizations (* in Fig 2A) erupted from the excitable gap adjacent to the SW tip, then the SW became nonstationary and began to meander widely in the myocardial tissue (1,300-1,400 ms). Subsequently, afterdepolarizations ( $\dagger$ in Fig $2 \mathrm{~A}$ ) also erupted from the excitable gap a little further from the SW tip, and part of the wavefront of the SW arm was disturbed by the afterdepolarization area, resulting in the first SW breakup $(1,430-1,450 \mathrm{~ms})$. In this manner, the SW meandering and occasional breakup occurred frequently because the afterdepolarizations randomly erupted from the excitable gap in the tissue; therefore, the wave dynamics degenerated into a chaotic state within a few seconds (1,500-3,000 ms).

Fig 3 shows the trajectory of the SW tip on the epicardial (front) tissue surface during the reentrant activation shown in Fig 2A. The SW tip initially rotated around the fixed small circular core (stationary reentry: 500-1,260 ms), but later the SW tip had an asymmetric and random (not the symmetric petal type) trajectory (meandering: from 1,265 $\mathrm{ms}$ ), even though the simulated myocardial tissue was completely homogeneous and isotropic. Fig 3 also clearly shows the SW tip trajectory during the SW breakup (wave breakup: from $1,430 \mathrm{~ms}$ ).

To examine the role of DADs and phase-3 EADs during SW reentry, we mathematically blocked calcium ion release from the junctional SR to the myoplasm (Irel), which directly induced the DADs and phase-3 EADs. This modification did not alter the APD restitution curve (not shown).

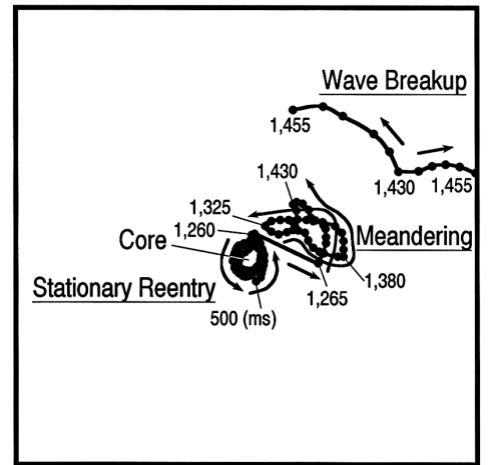

Fig 3. The trajectory of the spiral wave (SW) tip on the epicardial (front) tissue surface shown in Fig 2A. Large square shows the border of the epicardial tissue surface. Black solid circles represent the location of the SW tip by 5 -ms steps, and black solid lines connecting the black solid circles represent the trajectory of the SW tip. Black solid arrows denote the direction of the meandering and drifting of the SW tip. Numbers beside the black solid circles indicate the simulation time (ms).

As shown in Fig 2B, during the whole simulation period (to $3,000 \mathrm{~ms}$ ) there was a stationary SW reentry with neither meandering nor breakup because of the inhibition of the afterdepolarizations.

The simulated ECGs calculated from the transmembrane potential maps during the SW reentries are shown in Fig 2C. In the Irel(-) (modified) model, the simulated ECG showed rapid regular and uniform QRS morphology during the whole simulation period (to $3,000 \mathrm{~ms}$ ). On the other hand, in the Irel(+) (original) model, the simulated ECG initially also showed rapid regular and uniform QRS morphology; however, following the first eruption of the afterdepolarizations, they showed an irregular and multiform QRS morphology (1,250-3,000 ms), which may correspond to that shown on a clinical ECG during the transition from VT to VF.

\section{DADs and Phase-3 EADs During SW Reentry}

The tracings in Fig 4 show the transmembrane potential $(\mathrm{Vm})$ and the detailed intracellular calcium dynamics
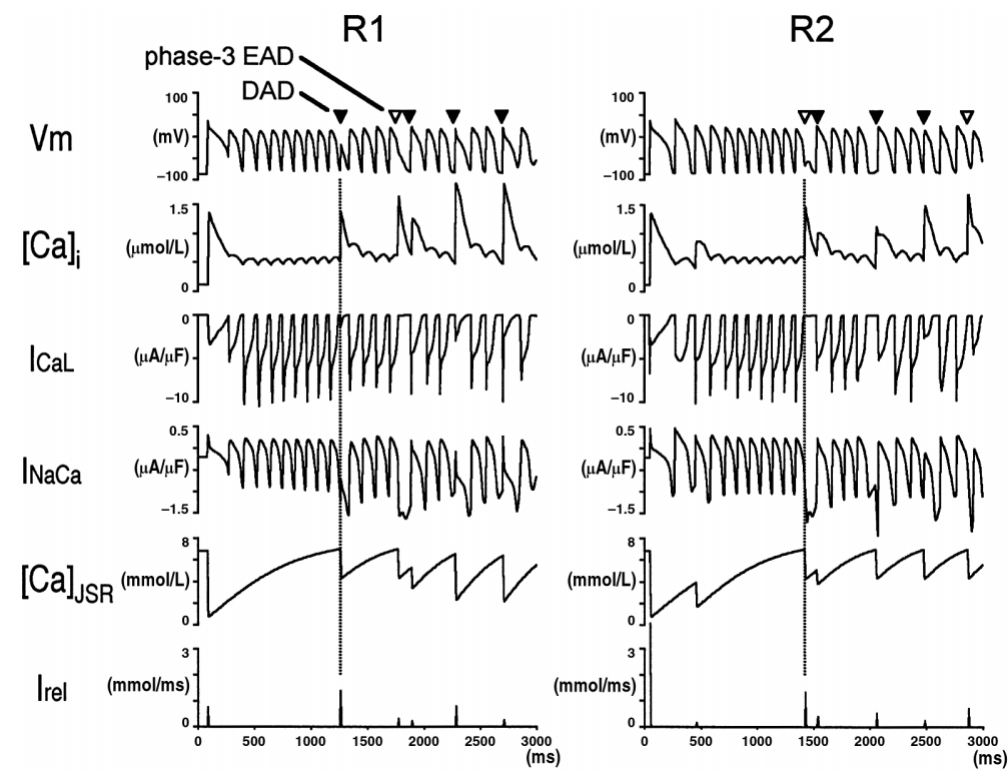

Fig 4. These traces show the transmembrane potential $(\mathrm{Vm})$, the intracellular calcium concentration ([Ca]i), the L-type calcium channel current (ICaL), the sodium-calcium exchanger current $(\mathrm{INaCa})$, the calcium concentration of the junctional SR ([Ca]JSR), and the calcium release from the junctional sarcoplasmic reticulum to the myoplasm (Irel). These data were recorded at R1 and R2 in Fig 2A. Dotted vertical lines represent the timing of the first delayed afterdepolarization (DAD; black solid triangle) and phase-3 early afterdepolarization (phase-3 EAD; black open triangle). The dotted vertical line in each panel represents the time that the first DAD or phase-3 EAD was induced in each myocardial unit. See text for details. 


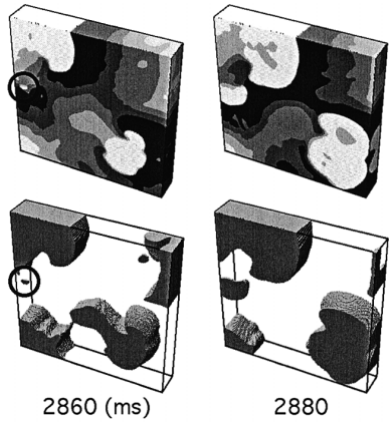

Fig 5. An example of breakthrough wave in the 3-dimensional cardiac tissue during the spiral wave reentry. The excitation waves in the upper panel are displayed as a gray scale as described in the legend for Fig 2, and those in the lower panel in gray represent voltage greater than $-25 \mathrm{mV}$.

during the SW reentry obtained from 2 myocardial units $\left[\mathrm{R} 1\left(^{*}\right)\right.$ and R2( $\left.\left.\dagger\right)\right]$ shown in Fig 2A. Each myocardial unit was rapidly excited during the SW reentry (cycle length was approximately $110 \mathrm{~ms}$ ), which was sufficient to cause intracellular calcium overload conditions, especially junctional SR calcium overload ( $>7 \mathrm{mmol} / \mathrm{L})$, and this activated the Irel. This Irel elicited DAD and phase-3 EAD without or with quite a few ICaL and INaCa enhancements at the excitable gap. As shown in the left panel of Fig 4, the first solid triangle represents a partial depolarization elicited by DAD in response to Irel. The first open triangle represents a delay of terminal repolarization of the preceding action potential because of phase-3 EAD. The second to fourth solid triangles represent the full action potentials elicited by DADs in response to Irel. As shown in the right panel of Fig 4, the first open triangle represents the delay of terminal repolarization because of phase-3 EAD. The first to third solid triangles represent the full action potentials elicited by DADs. The second open triangle represents the full action potential elicited by phase-3 EAD. Thus, the DADs and phase-3 EADs mostly induced TA; however, it is noteworthy that these afterdepolarizations contributed as a refractory area that was able to cause a local conduction block, even though these afterdepolarizations remained subthreshold (refer to the 1,430-1,450 ms panels of Fig 1A, and the corresponding time in the R2 panel of Fig 4). When the local conduction block occurred near the SW tip (eg, first DAD at $1,250 \mathrm{~ms}$ in the R1 panel) and at the SW arm (eg, first phase-3 EAD at 1,430 ms in the R2 panel), the SW began to meander and to breakup, respectively. In addition, we found that small TA and afterdepolarizations only caused transient wave breakup and the resulting waves immediately merged into one wavefront (not shown). These results suggest that the size of the afterdepolarization area is one of the important factors involved in SW breakup.

\section{Breakthrough Waves During SW Reentry}

The mechanisms of breakthrough waves on the myocardial surface during VF remain unclear, although it has been reported that they may derive from intramural reentry resulting from complex geometry, ${ }^{23}$ rotational anisotropy,2 Purkinje-muscle junctions ${ }^{24}$ and intramyocardial Purkinje fibers ${ }^{25}$ In contrast, as mentioned briefly in a previous study, ${ }^{26}$ our simulations (see upper panel of Fig 5) also suggested that the afterdepolarizations are another important factors in the mechanism of breakthrough waves during VF. For example, the lower panel of Fig 5 (skeleton view) clearly shows that the breakthrough wave (black open circle) was not the result of an intramural reentry. This afterdepolarization mechanism of breakthrough waves does not depend on the myocardial wall thickness; therefore, we conjecture that the concept of afterdepolarizations as a factor in the mechanism of breakthrough wave can be extended to a possible role of afterdepolarizations during atrial tachycardia and fibrillation.

\section{Discussion}

The major findings of the present study are (1) DADs and phase-3 EADs may play important roles in the mechanisms of 3-D SW meandering and breakup; (2) the mechanisms were independent of the APD restitution curve; (3) TA and afterdepolarizations that remained subthreshold caused local conduction block of wavefronts; and (4) local conduction block adjacent to and far from the SW tip resulted in SW meandering and breakup, respectively.

\section{Conditions of DADs and Phase-3 EADs}

Afterdepolarizations (DADs and phase-3 EADs) occur under a variety of conditions that cause an increase in the calcium ion concentration of the myoplasm and SR above normal levels; that is, calcium overload; digitalis toxicity with a greater rate of stimulation, ${ }^{10,27-29}$ catecholamines ${ }^{30-32}$ quinidine, 33 myocardial hypertrophy 34 myocardial infarction $^{35}$ etc. Because of these apparently ionic mechanisms of afterdepolarizations and calcium overload with reentrant activation or some pathologic abnormalities, it is plausible that DADs and phase-3 EADs may be induced by repetitive excitation during SW reentry and play important roles in the degeneration into a chaotic state.

\section{Afterdepolarization Mechanism is Independent of Restitution Properties}

Fig 1 and 2A-C clearly show that the mechanisms of SW meandering and breakup resulting from the DADs and phase-3 EADs were independent of the APD restitution curve. The SW instability can be simply explained by the collision between the wavefront of the SW arm and the refractory area resulting from the afterdepolarizations. In addition, we extended this afterdepolarization mechanism concept to the generation of breakthrough waves that may cause complexity during SW reentry. That is to say, the afterdepolarizations may cause SW instability even if the APD restitution slope is gentle $(<1)$. This is a quite interesting and important viewpoint for the production of new antiarrhythmic agents.

Antiarrhythmic agents for VF should be investigated based on 2 strategies: The prevention of (1) the initiation of VT by restraining the triggering events and/or altering the properties of reentrant circuits, and (2) the transition from VT to VF. It has been generally considered that suppressing the afterdepolarizations causing TA is effective only for the former strategy, but it was recently suggested that flattening the APD restitution curve might be effective for the latter ${ }^{6,7}$ We also conceded that a flattened APD restitution curve is effective for the latter strategy even in 3-D tissue with rotational anisotropy 22 On the other hand, in the present study we found that restraining afterdepolarizations is indispensable for both strategies, and our results suggest that only altering the restitution properties is insufficient to stabilize the SW reentry. We would like to stress that the reducing afterdepolarizations and TA (as with the use of 
agents such as lidocaine, ${ }^{36}$ doxorubicin, ${ }^{37}$ and calcium blocking agent ${ }^{12}$ ) may be important as well for the latter strategy.

\section{Comparison With Previous Studies}

Chudin et al 38,39 computationally studied the stability of 2-dimensional (2-D) SW reentry using a modified LRd model. They focused on the intracellular calcium dynamics, and found that the instability in the intracellular calcium dynamics during SW reentry amplified $\mathrm{INaCa}$ and prolonged APD, leading to electrophysiologic inhomogeneities and SW instability. This finding is generally compatible with our data (Fig 4); however, they did not study either the 3-D effect or the simulated ECG and, moreover, they did not discuss any strategies for the treatment of VF.

More recently, Pollard et al ${ }^{40}$ also simulated SW reentry in 2-D myocardial tissue with the LRd model and focused on, as in our study, not only the intracellular calcium dynamics but also the mechanisms of SW meandering and breakup resulting from the afterdepolarizations. Their findings ${ }^{40}$ show good agreement with ours ${ }^{41}$ but they did not discuss the 3-D effect or the simulated ECG, which we have focused on in this study. Furthermore, they did not discuss the mechanism of the afterdepolarizations from a clinical viewpoint.

\section{Study Limitations}

There were several important limitations in the present study. Although the LRd model is one of the latest versions of the mammalian ventricular cell model and can simulate dynamic changes in ionic concentrations, it is based mainly on single-cell data from the guinea pig. Therefore, we know that this mathematical model is not completely concordant with the human physiologic myocardial cell, and that the afterdepolarizations do not occur as easily in physiologic conditions. For example, the critical cycle length causing irregular calcium oscillation leading to periodic Irel was greater than $500 \mathrm{~ms}$, but this may not be an actual physiological condition. As another example, Riccio et $\mathrm{al}^{42,43}$ reported that the dynamic restitution slope recorded during rapid pacing and during VF was more than 1 ; however, the dynamic restitution slope with the present mathematical model was less than 1 . Moreover, we did not use the updated LRd model ${ }^{44}$ because we did not focus on potassium currents. From a structural point of view, we did not consider the spatial heterogeneity in refractoriness ${ }^{45}$ and constructed a homogeneous model with a monodomain nature instead of a bidomain nature ${ }^{46}$ because of the present limitations in computer performance and the finite amount of memory. Despite these limitations, we speculate that the DADs and phase-3 EADs are important mechanisms in the degeneration into VF. Additional studies are required to ensure that our findings are valid in a variety of myocardial cell models, and further experimental studies will be needed to elucidate the real mechanisms of the transition from VT to $\mathrm{VF}$.

\section{Conclusion}

Our simulation studies suggested that the DADs and phase-3 EADs may play important roles in the transition from VT to VF, and that these mechanisms are independent of the APD restitution curve. Our findings are supported by the fact that VF is more likely to occur in conditions where there is intracellular calcium overload causing afterdepo- larizations.

\section{Acknowledgments}

This study was supported in part by Grant-in-Aid for Scientific Research (A) from The Ministry of Education, Science, Sports and Culture (12308046); Grant-in-Aid for Scientific Research (C) from The Ministry of Education, Science, Sports and Culture (12670660); Grant-in-Aid for Scientific Research (C) from The Ministry of Education, Science, Sports and Culture (12670698); Grant-in-Aid for Research and Development for Applying Advanced Computational Science and Technology (12B-1); and the Halberg Prize of the 2nd International Symposium, Workshop on Chronoastrobiology and Chronotherapy to Dr Ashihara.

\section{References}

1. Davidenko JM, Pertsov AV, Salomonz R, Baxter W, Jalife J. Stationary and drifting spiral waves of excitation in isolated cardiac muscle. Nature 1992; 355: 349-351.

2. Gray RA, Jalife J, Panfilov AV, Baxter WT, Cabo C, Davidenko JM, et al. Mechanisms of cardiac fibrillation. Science 1995; 270: $1222-$ 1225.

3. Pratt CM, Francis MJ, Luck JC, Wyndham CR, Miller RR, Quinones MA. Analysis of ambulatory electrocardiograms in 15 patients during spontaneous ventricular fibrillation with special reference to preceding arrhythmic events. J Am Coll Cardiol 1983; 2: 789-797.

4. Karma A. Electrical alternans and spiral wave breakup in cardiac tissue. Chaos 1994; 4: 461-472.

5. Davidenko JM, Salomonsz R, Pertsov AM, Baxter WT, Jalife J. Effects of pacing on stationary reentrant activity: Theoretical and experimental study. Circ Res 1995; 77: 1166-1179.

6. Qu Z, Weiss JN, Garfinkel A. Cardiac electrical restitution properties and stability of reentrant spiral waves. Am J Physiol 1999; 276: $\mathrm{H} 269-\mathrm{H} 283$.

7. Weiss JN, Garfinkel A, Karagueuzian HS, Qu Z, Chen PS. Chaos and the transition to ventricular fibrillation: A new approach to antiarrhythmic drug evaluation. Circulation 1999; 99: 2819-2826.

8. Reiter M. Die Entstehung von 'Nachkontraktionen' im Herzmuskel unter Einwirkung von Calcium und von Digitalisglykosiden in Abhängigkeit von der Reizfrequenz. Arch Exp Pathol Pharmakol 1962; 242: $497-507$.

9. Cranefield PF. Action potentials, afterpotentials and arrhythmias. Circ Res 1977; 41: 415-423.

10. Zipes DP, Arbel E, Knope RF, Moe GK. Accelerated cardiac escape rhythms caused by ouabain intoxication. Am J Cardiol 1974; 33: $248-253$.

11. Zipes DP, Foster PR, Troup PJ, Pedersen DH. Atrial induction of ventricular tachycardia: Reentry versus triggered automaticity. Am J Cardiol 1979; 44: $1-8$.

12. Vos MA, Gorgels APM, Leunissen JDM, Wellens HJJ. Flunarizine allows differentiation between mechanisms of arrhythmias in the intact heart. Circulation 1990; 81: 343-349.

13. Sato T, Hirao K, Hiejima K. The relationship between early afterdepolarization and the occurrence of torsades de pointes: An in vivo canine model study. Jpn Circ J 1993; 57: 543-552.

14. Ishida $\mathrm{S}$, Ito M, Takahashi N, Fujino $\mathrm{T}$, Akimitsu $\mathrm{T}$, Saikawa $\mathrm{T}$. Caffeine induces ventricular tachyarrhythmias possibly due to triggered activity in rabbits in vivo. Jpn Circ J 1996; 60: 157-165.

15. Shimoike E, Ueda N, Maruyama T, Kaji Y, Kanaya S, Fujino T, et al. Heart rate variability analysis of patients with idiopathic left ventricular outflow tract tachycardia: Role of triggered activity. Jpn Circ J 1999; 63: 629-635.

16. Luo $\mathrm{CH}$, Rudy Y. A dynamic model of the cardiac ventricular action potential. II. Afterdepolarizations, triggered activity, and potentiation. Circ Res 1994; 74: 1097-1113.

17. Xu J, Zaim S, Pelleg A. Effects of pinacidil, verapamil, and heart rate on afterdepolarizations in the guinea-pig heart in vivo. Heart Vessels 1996; 11: 289-302.

18. Fabiato A, Fabiato F. Contraction induced by a calcium-triggered release of calcium from the sarcoplasmic reticulum of single skinned cardiac cells. J Physiol 1975; 249: 469-495.

19. Fabiato A. Simulated calcium current can both cause calcium loading in and trigger calcium release from the sarcoplasmic reticulum of a skinned canine cardiac Purkinje cell. J Gen Physiol 1985; 85: 291 320.

20. Luo CH, Rudy Y. A dynamic model of the cardiac ventricular action potential. I. Simulations of ionic currents and concentration changes. Circ Res 1994; 74: 1071-1096.

21. Jorgensen AO, Broderick R, Somlyo AP, Somlyo AV. Two struc- 
turally distinct calcium storage sites in rat cardiac sarcoplasmic reticulum: An electron microprobe analysis study. Circ Res 1988; 63: $1060-1069$.

22. Ashihara T, Namba T, Ikeda T, Ito M, Kinoshita M, Nakazawa K. Breakthrough waves during ventricular fibrillation depend on the degree of rotational anisotropy and the boundary conditions: A simulation study. J Cardiovasc Electrophysiol 2001; 12: 312-322.

23. Ashihara T, Namba T, Ito M, Kinoshita M, Nakazawa K. The dynamics of vortex-like reentry wave filaments in three-dimensional computer models. J Electrocardiol 1999; 32(Suppl): 129-138.

24. Berenfeld O, Jalife J. Purkinje-muscle reentry as a mechanism of polymorphic ventricular arrhythmias in a 3-dimensional model of the ventricles. Circ Res 1998; 82: 1063 - 1077.

25. Valderrábano M, Lee M-H, Ohara T, Lai AC, Fishbein MC, Lin S-F, et al. Dynamics of intramural and transmural reentry during ventricular fibrillation in isolated swine ventricles. Circ Res 2001; 88: 839-848.

26. Pogwizd SM. Nonreentrant mechanisms underlying spontaneous ventricular arrhythmias in a model of nonischemic heart failure in rabbits. Circulation 1995; 92: 1034-1048.

27. Davis LD. Effect of changes in cycle length on diastolic depolarization produced by ouabain in canine Purkinje fibers. Circ Res 1973; 32: $206-214$.

28. Ferrier GR, Saunders JH, Mendez C. A cellular mechanism for the generation of ventricular arrhythmias by acetylstrophanthidin. Circ Res 1973; 32: 600-609.

29. Rosen MR, Gelband H, Merker C, Hoffman BF. Mechanisms of digitalis toxicity: Effects of ouabain on phase four of canine Purkinje fiber transmembrane potentials. Circulation 1973; 47: 681-689.

30. Carmeliet E, Vereeche J. Adrenaline and the plateau phase of the cardiac action potential. Pfluegers Arch 1969; 313: 300-315.

31. Belardinelli L, Isenberg G. Actions of adenosine and isoproterenol on isolated mammalian ventricular myocyte. Circ Res 1983; 53: 287-297.

32. Priori SG, Corr PB. Mechanisms underlying early and delayed afterdepolarizations induced by catecholamines. Am J Physiol 1990; 258: H1796-H1805.

33. Wit AL, Tseng GN, Henning B, Hanna MS. Arrhythmogenic effects of quinidine on catecholamine-induced delayed afterdepolarizations in canine atrial fibers. J Cardiovasc Electrophysiol 1990; 1: 15-30.

34. Aronson RS. Afterpotentials and triggered activity in hypertrophied myocardium from rats with renal hypertension. Circ Res 1981; 48:
$720-727$.

35. Dangman KH, Dresdner KP, Zaim S. Automatic and triggered impulse initiation in canine subepicardial ventricular muscle cells from border zones of 24-hour transmural infarcts: New mechanism for malignant cardiac arrhythmias? Circulation 1988; 78: 1020-1030.

36. Sheu SS, Lederer WJ. Lidocaine's negative inotropic and antiarrhythmic actions: Dependence on shortening of action potential duration and reduction of intracellular sodium activity. Circ Res 1985; 57: $578-590$.

37. LeMarec H, Spinelli W, Rosen MR. The effects of doxorubicin on ventricular tachycardia. Circulation 1986; 74: 881-889.

38. Chudin E, Garfinkel A, Weiss J, Karplus W, Kogan B. Wave propagation in cardiac tissue and effects of intracellular calcium dynamics (computer simulation study). Prog Biophys Mol Biol 1998; 69: 225236.

39. Chudin E, Goldhaber J, Garfinkel A, Weiss J, Kogan B. Intracellular $\mathrm{Ca}^{2+}$ dynamics and the stability of ventricular tachycardia. Biophys $J$ 1999; 77: 2930-2941.

40. Pollard AE, Lit LM, Rogers JM. Functional reentry's influence on intracellular calcium in the LRd membrane equations. IEEE Trans Biomed Eng 2000; 47: 1228-1236.

41. Ashihara T, Inagaki M, Toda S, Namba T, Ikeda T, Kawase A, et al. DADs play an important role in the transition from VT to VF: A simulation study employing a Luo-Rudy II model (abstract). Jpn Circ $J$ 2001; 65(Suppl I-A): 135.

42. Koller ML, Riccio ML, Gilmour RF Jr. Dynamic restitution of action potential duration during electrical alternans and ventricular fibrillation. Am J Physiol 1998; 275: H1635-H1642.

43. Riccio ML, Koller ML, Gilmour RF Jr. Electrical restitution and spatiotemporal organization during ventricular fibrillation. Circ Res 1999; 84: 955-963.

44. Zeng J, Laurita KR, Rosenbaum DS, Rudy Y. Two components of the delayed rectifier $\mathrm{K}+$ current in ventricular myocytes of the guinea pig type: Theoretical formulation and their role in repolarization. Circ Res 1995; 77: 140-152.

45. Namba T, Ashihara T, Nakazawa K, Ohe T. Spatial heterogeneity in refractoriness as a proarrhythmic substrate: Theoretical evaluation by numerical simulation. Jpn Circ J 2000; 64: 121-129.

46. Ashihara T, Yao T, Namba T, Ito M, Ikeda T, Kawase A, et al. Electroporation in a model of cardiac defibrillation. $J$ Cardiovasc Electrophysiol 2001; 12: 1393-1403. 